\title{
地黄对阿尔茨海默病小鼠行为学的影响 及其对血脑屏障的保护作用
}

赵大鹏, 陆韵薇, 于顾然

南京中医药大学附属医院神经内科, 江苏南京 210029

[摘 要] 目的: 探讨中药地黄对阿尔茨海默病模型小鼠日常活动能力和恐惧记 忆能力的行为学影响及其对血脑屏障的保护作用。方法: 38 只 4 月龄 APP/PS1 双转 基因小鼠随机分为模型对照组 (给予等渗氯化钠溶液)、地黄小剂量组(给予地黄 $5 \mathrm{~g} \cdot \mathrm{kg}^{-1} \cdot \mathrm{d}^{-1}$ ) 和地黄大剂量组 (给予地黄 $12 \mathrm{~g} \cdot \mathrm{kg}^{-1} \cdot \mathrm{d}^{-1}$ ), 连续灌胃给药 3 个月, 每日给药 1 次。分别采用筑巢实验和条件恐惧实验检测小鼠日常活动能力和恐惧 记忆能力; 硫黄素 $\mathrm{T}$ 染色检测小鼠皮层和海马 CA1 区 $\beta$ 淀粉样蛋白 $(\mathrm{A} \beta$ ) 沉积; 免疫 荧光双重标记染色检测小鼠皮层和海马 CA1 区血管内皮完整性及血脑屏障渗出情 况。结果: 与模型对照组比较, 地黄小、大剂量组日常活动能力均明显改善 (均 $P<0.01)$; 地黄大剂量组恐惧记忆能力明显提高 $(P<0.01)$; 地黄大剂量组皮层和海 马 CA1 区 A 3 沉积量明显减少, 面积占比明显减小, 地黄小剂量组海马 CA1 区 A 3 沉 积面积占比也减小 (均 $P<0.05)$; 地黄小、大剂量组大脑皮层 CD34 阳性面积占比显 著增加, 纤维蛋白原阳性面积占比显著减小 $($ 均 $P<0.05)$; 地黄大剂量组海马 CA1 区 $\mathrm{CD} 34$ 阳性面积占比显著增加, 纤维蛋白原阳性面积占比显著减小 $($ 均 $P<0.05)$ 。结 论: 中药地黄可以提高模型小鼠的日常活动能力及恐惧记忆功能, 减少脑内 $A \beta$ 沉 积, 且大剂量时效果更为显著; 其机制可能与降低血脑屏障通透性、保护血脑屏障 完整性有关。

[关键词] 阿尔茨海默病; 地黄;日常活动能力; 条件性恐惧记忆; $\beta$ 淀粉样蛋白; 血 脑屏障; 转基因小鼠

[中图分类号 ］R285［文献标志码 ］A

\section{Effects of Radix Rehmanniae on behavior and blood-brain barrier in Alzheimer's disease mice}

ZHAO Dapeng, LU Yunwei, YU Guran (Department of Neurology, Affiliated Hospital of Nanjing University of Chinese Medicine, Nanjing 210029, China)

Corresponding author: YU Guran, E-mail:dr.ygrdf@163.com, https://orcid.org/0000-

收稿日期:2021-03-03 接受日期:2021-07-05 网络预发表日期:2021-10-22

基金项目:国家自然科学基金(81573771)

第一作者: 赵大鹏, 博士研究生, 主要从事神经退行性变性疾病的中西医结合治疗研究;E-mail :zhaodapeng0302@163. com; https://orcid.org/0000-0003-0588-3183

通信作者:于顾然,教授,博士生导师,主要从事神经退行性变性疾病的中西医结合治疗研究;E-mail:dr.ygrdf@163.com; https ://orcid.org/0000-0002-9356-8695 
0002-9356-8695

[ Abstract ] Objective: To investigate the effects of Radix Rehmanniae on behavior and blood brain barrier (BBB) in Alzheimer's disease mice. Methods: Thirty-eight 4-month-old APP/PS1 double transgenic mice were randomly divided into three groups: model group, Radix Rehmanniae low-dose group and Radix Rehmanniae high-dose group. Saline, Radix Rehmanniae $5 \mathrm{~g} \cdot \mathrm{kg}^{-1} \cdot \mathrm{d}^{-1}$ and Radix Rehmanniae $12 \mathrm{~g} \cdot \mathrm{kg}^{-1} \cdot \mathrm{d}^{-1}$ were given to each group by continuous gavage once a day for three months, respectively. The changes in activities of daily live and fear conditioning memory behavior of mice were examined by nesting behavior test and fear conditioning test, respectively. The $\beta$-amyloid protein (A $\beta$ ) depositions in cortex and hippocampal CA1 area of mice were detected by thioflavin T staining. The CD34 and activities fibrinogen (Fib) immunofluorescence double staining were used to determine the vascular endothelial integrity and BBB exudation. Results: Compared with model mice, activities of daily live were significantly improved in Radix Rehmanniae low-dose and high-dose groups (both $P<0.01$ ), the fear memory ability was significantly increased in Radix Rehmanniae high-dose group $(P<0.01)$. The amount of A $\beta$ deposition in cortex and hippocampal CA1 decreased significantly in Radix Rehmanniae high-dose group, the area ratio decreased significantly; the area ratio of $A \beta$ deposition in hippocampal CA1 region in Radix Rehmanniae low-dose group also decreased (all $P<0.05)$. The proportions of CD34 positive area of cortex in low and high dose Radix Rehmanniae groups increased, the percentage of fibrinogen positive area decreased (all $P<0.05)$. The proportion of CD34 positive area in hippocampal CA1 region in Radix Rehmanniae high-dose group was significantly increased, the percentage of fibrinogen positive area decreased significantly (both $P<0.05)$. Conclusions: Radix Rehmanniae, especially high-dose can improve the activities of daily live and fear conditioning memory function of APP/PS1 mice, reduce the deposition of A $\beta$ in brain. The mechanism may be related to the reduction of $\mathrm{BBB}$ permeability and the protection of the integrity of $\mathrm{BBB}$.

[ Key words ] Alzheimer's disease; Radix Rehmanniae; Activity of daily live; Fear conditioning memory; Amyloid $\beta$-protein; Blood-brain barrier; Transgenic mice

[J Zhejiang Univ (Med Sci), 2021, 50(5): 553-560.]

[ 缩略语 ] 阿尔茨海默病 (Alzheimer's disease, $\mathrm{AD})$; 4', 6-二脒基-2-苯基吲哚 $\left(4^{\prime}, 6\right.$ dinidino-2-phenylindole,DAPI); $\beta$ 淀粉样蛋白 (amyloid $\beta$-protein, A $\beta$ )

$\mathrm{AD}$ 是一种以进行性认知损害和性格改变为 特征的神经退行性变性疾病, 日常活动能力受损 及行为精神症状的出现往往给 $\mathrm{AD}$ 患者及照料者 带来沉重的负担。目前治疗 AD 的常规药物并不 能阻断疾病进程, 因此寻找一种新的药物已成为 临床研究的热点 ${ }^{[1]}$ 。祖国医学有着简便效廉的特 点, 且中药具有多靶点、不良反应小等优势, 受到 广泛关注。AD属于祖国医学中 “痴呆” 的范畴,
其基本病机为髓海不足、神机失用,其根本多为肾 精亏虚、髓楲脑消, 故治疗也多用补虚扶正、充髓 养脑的方法 ${ }^{[2]}$ 。在众多补肾益精药物中, 地黄为 古今医家所重用 ${ }^{[3]}$, 《神农本草经》描述其能 “逐血痹, 填骨髓, 长肌肉”。

血脑屏障是血液与脑组织间的特殊屏障, 可 以阻止有害物质进人脑内损伤神经元。血脑屏障 损伤是 $\mathrm{AD}$ 等神经退行性变性疾病患者脑部重要 
的病理特征之一 ${ }^{[4]}$ 。因此,保护血脑屏障具有重 要意义。本团队前期的体外研究结果显示, 以地黄 为君药的中药复方 “补肾益精方” 的含药血清以 及地黄主要成分梓醇对血脑屏障均有保护作 用 ${ }^{[5-6]}$, 且单味中药地黄的水煎剂也具有显著的神 经保护功效 ${ }^{[7]}$ 。本研究进一步就单味中药地黄改 善APP/PS1 模型小鼠的日常活动能力和恐惧记忆 能力等行为学进行观察, 并探讨其在体内对血脑 屏障的保护作用。

\section{1 材料与方法}

\section{1 动物、试剂及仪器}

38 只 4 月龄雄性 $\mathrm{APP} / \mathrm{PS} 1$ 双转基因 $\mathrm{AD}$ 模型 小鼠B6C3-Tg (APPswe/PSEN1dE9)购自江苏集萃 药康生物科技股份有限公司, 许可证号为 SCXK (苏)2018-0008。小鼠饲养于南京中医药大学无 特定病原体级的动物实验屏障系统中, $12 \mathrm{~h}$ 光暗交 替, 温度为 $(22 \pm 2)^{\circ} \mathrm{C}$, 湿度为 $(50 \pm 5) \%$, 自由饮 水和摄食。所有实验程序均经南京中医药大学动 物伦理委员会审批,并严格按照美国《实验动物 饲养管理和使用指南》 ${ }^{[8]}$ 执行。

中药地黄颗粒 (生、熟地黄比例 1:1) 为江阴 天江药业有限公司产品 (批号 18112551); 硫黄素 T 为上海源叶生物科技有限公司产品; 免抗纤维蛋 白原 $\alpha$ 链单克隆抗体、鼠抗 CD34单克隆抗体、山羊 抗兔二抗、山羊抗大鼠二抗为英国 Abcam 公司产 品; Nestleta棉花压片 $5 \mathrm{~cm} \times 5 \mathrm{~cm}$ 为南京何苗生物 技术有限公司产品; ACH-100 Coulbourn 恐惧条件 系统为美国 Coulbourn Instruments 产品; 莫里斯水 迷宫视频分析系统为安徽正华生物仪器设备有限 公司产品; Nikon ECLIPSE Ni 苂光正置显微镜为日 本尼康公司产品。

\section{2 实验分组及取材}

4 月龄 $\mathrm{AD}$ 模型小鼠用随机数字表随机分为 模型对照组 (13 只)、地黄小剂量组 (12 只)和地黄 大剂量组 (13 只)。地黄颗粒溶解于等渗氯化钠溶 液中, 根据中药药理学通用粗略换算公式 ${ }^{[9]}$, 地黄 小剂量组给予地黄 $5 \mathrm{~g} \cdot \mathrm{kg}^{-1} \cdot \mathrm{d}^{-1}$, 地黄大剂量组 给予地黄 $12 \mathrm{~g} \cdot \mathrm{kg}^{-1} \cdot \mathrm{d}^{-1}$ 。模型对照组给予等渗 氯化钠溶液。各组均灌胃给药 3 个月, 至小鼠 7 月 龄时行筑巢实验、条件恐惧实验, 再将小鼠以颈椎 脱臼法处死后断头取脑, 于左侧大脑半球前囟 $-1.70 \sim 2.30 \mathrm{~mm}$ 以 $5 \mu \mathrm{m}$ 的厚度进行冠状位连续切
片。每组随机选取 6 只小鼠的大脑切片行染色 分析。

\section{3 筑巢行为实验检测模型小鼠日常活动能力}

模型对照组、地黄小剂量组、地黄大剂量组各 有 $9 、 12$ 和 13 只小鼠进行筑巢行为实验, 具体方法 参照文献 $[10]$ 。测试第 1 天于夜节律开始前 $2 \mathrm{~h}$ (约 17:00) 将 $5 \mathrm{~cm} \times 5 \mathrm{~cm}$ 的 2 张压缩棉放置于笼中, 分别于 $2 、 24 、 48 \mathrm{~h}$ 按照文献 [11]中的评分标准进 行评分。结果采用盲法打分,并统计小鼠的筑巢 分数。

1.4 条件恐惧实验检测模型小鼠恐惧记忆能力

模型对照组、地黄小剂量组、地黄大剂量组各 有 $7 、 10$ 和 12 只小鼠进行条件恐惧实验。第 1 天为 恐惧记忆形成。将小鼠放人实验箱中, 关闭箱 门。开启条件恐惧系统软件进行训练: 首先小鼠 在箱内自由活动 $3 \mathrm{~min}$ 以适应环境; 然后开启声音 刺激 (Tone 音, $2.5 \mathrm{~Hz}, 85 \mathrm{~dB}$ ), 持续 $30 \mathrm{~s}$; 紧跟电刺 激 $(0.75 \mathrm{~mA})$, 持续 $2 \mathrm{~s}$; 接着无声音刺激和电刺激 2 min。以上声音刺激一电刺激一无任何刺激过程 重复6次 (共7次,时长 $1244 \mathrm{~s}$ )。第 2 天为恐惧记忆 关联期, 将小鼠放人实验箱中, 关闭箱门。去掉声 音刺激和电刺激, 小鼠自由活动, 时长 $1200 \mathrm{~s}$ 。第 3 天进行恐惧记忆测试。在试验箱对角线加上纸板 以改变之前的实验环境, 在栅栏上放上纸板,使小 鼠脚底触觉发生变化。设置测试条件: 恐惧箱内 室灯打开,放人小鼠自由活动并适应 $3 \mathrm{~min}$; 开始 声音刺激 $(2.5 \mathrm{~Hz}, 85 \mathrm{~dB}$ ), 持续 $30 \mathrm{~s}$; 停止 $2 \mathrm{~min}$ 不 给予任何刺激;再次给予声音刺激。整个过程共 进行 10 次, 总时长为 $1680 \mathrm{~s}$, 实验过程中保证外部 环境安静。统计各组僵直时间百分比。

\section{5 硫磺素 $\mathrm{T}$ 染色观察模型小鼠大脑 $\mathrm{A} \beta$ 沉积情况}

将小鼠大脑切片浸泡于多聚甲醛 $10 \mathrm{~min}$, 然 后置于磷酸盐缓冲液 (酸碱度为 7.4 ) 中在脱色摇 床上晃动洗涤 3 次,每次 5 min。用 $50 \%$ 乙醇配制 $0.3 \%$ 硫磺素 $\mathrm{T}$ 溶液, 用滤纸过滤, 室温孵育 $8 \mathrm{~min}$ 。将玻片置于磷酸盐缓冲液中在脱色摇床上 晃动洗涤 3 次, 每次 5 min。切片甩干后滴加DAPI 染液, 室温避光孵育 $10 \mathrm{~min}$ 。最后, 用抗荧光淬灭 封片剂封片。将制备好的硫磺素 $\mathrm{T}$ 染色切片置于 苂光显微镜下用 40 倍视野观察并采集图像。每张 切片随机选取皮层 2 个视野, 海马 CA 1 区 1 个视 野, 应用ImageJ 软件分析每个视野中 $A \beta$ 沉积量和 沉积面积占比(阳性总面积/统计场总面积)。 
1.6 免疫苂光双重标记染色观察血脑屏障渗出 情况

将小鼠大脑切片浸泡于多聚甲醛中 $10 \mathrm{~min}$, 然后置于磷酸盐缓冲液中在脱色摇床上晃动洗涤 3 次, 每次 5 min。滴加山羊血清 $50 \mu \mathrm{L}$, 室温孵 育 $20 \mathrm{~min}$, 封闭非特异性结合位点。滴加一抗 (1：100稀释) $50 \mu \mathrm{L}, 37^{\circ} \mathrm{C}$ 下湿盒孵育 $2 \mathrm{~h}$, 磷酸盐 缓冲液浸洗 3 次, 每次 $5 \mathrm{~min}$ 。滴加二抗 $50 \mu \mathrm{L}$, $37^{\circ} \mathrm{C}$ 下避光孵育 $1 \mathrm{~h}$, 磷酸盐缓冲液浸洗 3 次, 每 次 5 min。每张切片滴加DAPI染液50 100 $\mu \mathrm{L}$, 室 温避光放置 5 min。最后用抗苂光淬灭封片剂封 片。将制备好的切片置于苂光显微镜下用 200 倍 视野观察并采集图像。每张切片随机选取皮层 2 个视野, 海马 CA1 区 1 个视野观察, 应用 ImageJ 软 件分析每个视野中的阳性面积占比, 即阳性总面 积/统计场总面积。

\section{7 统计学方法}

采用 GraphPad Prism 5 软件进行统计分析。 正态分布的计量数据以均数 \pm 标准差 $(\bar{x} \pm s)$ 表 示, 采用单因素方差分析, 两两比较用最小差异法 检验; 非正态分布的计量数据以中位数 (上下四分 位数) $\left[M\left(Q_{1}, Q_{3}\right)\right]$ 表示, 采用秩和检验。 $P<0.05$ 为差异具有统计学意义。

\section{2 结 果}

2.1 地黄可以改善 $\mathrm{AD}$ 模型小鼠的日常活动能力 筑巢 $2 \mathrm{~h}$ 时,地黄小剂量组筑巢评分高于模型 对照组 $(P<0.05)$; 筑巢 $24 、 48 \mathrm{~h}$ 时, 地黄小、大剂量 组筑巢分数均高于模型对照组 (均 $P<0.01$ )。见 表 1 。结果提示, 地黄可以改善小鼠的日常活动 能力。

\section{2 地黄可以改善 $\mathrm{AD}$ 模型小鼠的恐惧记忆能力} 模型对照组、地黄小剂量组和地黄大剂量组 偪直时间百分比分别为 $(36.66 \pm 12.95) \%$ 、 (39. $65 \pm 13.74) \%$ 和 $(64.08 \pm 11.08) \%$, 其中地黄
大剂量组僵直时间百分比较模型对照组和地黄小 剂量组均增加 $($ 均 $P<0.01)$, 地黄小剂量组僵直时 间百分比较模型对照组也有所增加,但差异无统 计学意义 $(P>0.05)$ 。结果提示, 大剂量地黄可以 提高 $\mathrm{AD}$ 模型小鼠的恐惧记忆能力。

\section{3 地黄可以减少 $\mathrm{AD}$ 模型小鼠大脑 $\mathrm{A} \beta$ 沉积}

各组小鼠的皮层和海马 $\mathrm{CA} 1$ 区均可见 $\mathrm{A} \beta$ 沉 积。与模型对照组比较,地黄大剂量组皮层和海马 $\mathrm{CA} 1$ 区 $\mathrm{A} \beta$ 沉积量明显减少,面积占比均减小 (均 $P<0.05)$; 地黄小剂量组海马 CA1 区 $A \beta$ 沉积面积占 比减小 $(P<0.05)$ 。与地黄小剂量组比较, 地黄大剂 量组大脑皮层的 $\mathrm{A} \beta$ 沉积量明显减少 $(P<0.01)$ 。见 图 1 和表 2 。结果提示, 地黄可以促进 $A \beta$ 的清除, 减 少其在大脑中的沉积。

2.4 地黄可以保护 $\mathrm{AD}$ 模型小鼠血脑屏障内皮 完整、减少血脑屏障渗出

免疫荧光双重标记染色结果显示, 各组大脑 皮层和海马 CA1 区均可见 CD 34 及纤维蛋白原阳性 表达。与模型对照组比较, 地黄小、大剂量组大脑 皮层 CD34 阳性面积占比均增加, 纤维蛋白原阳性 面积占比均减小 $($ 均 $P<0.05)$; 地黄大剂量组海马 CA1 区 CD34 阳性面积占比显著增加, 纤维蛋白原 阳性面积占比显著减小 (均 $P<0.05)$ 。与地黄小剂 量组比较, 地黄大剂量组皮层 CD34 阳性面积占比 显著增加 $(P<0.01)$ 。见图 2 和表 3 。结果提示, 中 药地黄可以保护血脑屏障的完整性,减少血脑屏障 的渗出。

\section{3 讨 论}

祖国医学认为 “疾呆” 的基本病机为肾精亏 耗、脑髓失养、神机失用, 临床往往表现为呆俊愚 笨、智能低下。又因肾在志为恐, 肾虚亦表现为惊 恐焦虑, 严重影响日常生活功能, 故补肾是治疗痴 呆不可忽视的一面 ${ }^{[3]}$ 。中药地黄具有益精填髓的 作用, 《本草经疏》誉其为 “补肾家之要药,益阴

表 1 筑巢实验中各组小鼠不同时间段的评分比较

Table 1 Scores of nesting behavior in each group

\begin{tabular}{crccc} 
& \multicolumn{1}{c}{} & & {$\left[M\left(Q_{1}, Q_{3}\right)\right.$ 或 $\left.(\bar{x} \pm s)\right]$} \\
\hline 组 别 & $n$ & $2 \mathrm{~h}$ & $24 \mathrm{~h}$ & $48 \mathrm{~h}$ \\
\hline 模型对照组 & 9 & $0.000(0.000,0.500)$ & $0.500(0.250,1.000)$ & $1.333 \pm 1.031$ \\
地黄小剂量组 & 12 & $0.500(0.500,0.500)^{*}$ & $1.750(1.000,0.250)^{* *}$ & $2.458 \pm 0.620^{* *}$ \\
地黄大剂量组 & 13 & $0.500(0.000,0.500)$ & $1.500(1.000,0.250)^{* *}$ & $2.538 \pm 0.628^{* *}$ \\
\hline
\end{tabular}

与模型对照组比较, ${ }^{*} P<0.05,{ }^{* *} P<0.01$. 
血之上品” ，《本草纲目》中论述其可 “填骨髓, 长肌肉, 生精血, 补五脏之内伤不足, 通血脉, 利耳 目”。现代药理学研究显示, 地黄在治疗老年痴 呆方面具有抗氧自由基、改善细胞内钙失衡、保护 线粒体、改善神经递质功能、抑制细胞调亡、调节 神经内分泌等作用 ${ }^{[12]}$ 。但关于中药地黄对血脑屏 障保护性研究较少, 而 $\mathrm{AD}$ 的发病与血脑屏障的结 构及功能的损伤又密切相关 ${ }^{[13]}$, 因此研究血脑屏 障的保护作用对 $\mathrm{AD}$ 的防治具有重要意义。

皮层

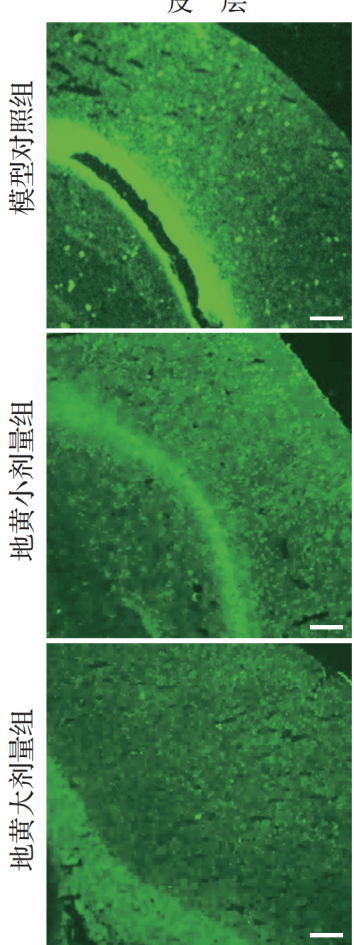

绿色苂光为 $\beta$ 淀粉样蛋白阳性染色. 模型对照组皮层和 海马CA1区 $\beta$ 淀粉样蛋白沉积量较多,地黄小剂量组和地黄 大剂量组皮层及海马CA1区 $\beta$ 淀粉样蛋白沉积量较模型对 照组减少, 尤以地黄大剂量组减少更为明显. 标尺 $=100 \mu \mathrm{m}$.

图 1 各组大脑皮层和海马CA1区 $\beta$ 淀粉样蛋白硫黄 素T染色结果

Figure 1 Thioflavin T staining of amyloid $\beta$-protein in cortex and hippocampus CA1 area
$\mathrm{APP} / \mathrm{PS} 1$ 双转基因小鼠是 $\mathrm{AD}$ 的有效评价模 型。该模型可以较好地模拟细胞外 $A \beta$ 沉积等 $A D$ 的神经病理学特征, 对 $\mathrm{AD}$ 的研究价值较大, 是目 前作为 $\mathrm{AD}$ 实验中最为常用的模型动物 ${ }^{[14]}$ 。日常 活动能力减退及记忆力下降严重影响了 $\mathrm{AD}$ 患者 的生活。本研究采用筑巢实验和条件恐惧实验分 别测试模型小鼠的日常活动能力和恐惧记忆能 力。筑巢是小鼠的日常行为, 其生理学基础复杂, 可受内侧前额叶皮质、海马等脑区的控制 ${ }^{[15-16]}$ 。 条件恐惧实验是基于经典的条件反射理论而建立 的, 即将一种条件性刺激和一种非条件性刺激相联 系, 以形成关联性学习记忆, 从而测定动物的学习、 不愉悦记忆经历和环境暗示之间关联的能力。条 件恐惧实验常被用来评价动物的条件性恐惧记 忆 ${ }^{[17]}$ 。本文资料显示, 中药地黄可以明显改善 $\mathrm{AD}$ 小鼠的日常活动能力和恐惧记忆能力。

$A D$ 的病因迄今不明, 研究发现与脑内 $A \beta$ 异 常沉积有关 ${ }^{[18]}$ 。在以往开展的各种临床和基础 研究也说明 $A \beta$ 是 $A D$ 发病的关键环节 ${ }^{[19]}$ 。A 级 联假说首次提出了以 $\mathrm{A} \beta$ 为主要成分的老年斑在 细胞外沉积可能是 $\mathrm{AD}$ 发病机制的主要启动因 素 ${ }^{[20]}$ 。大部分 $A D$ 患者脑内 $A \beta$ 升高是由脑组织对 $A \beta$ 清除障碍所引起。美国学者发现纤维性 $A \beta$ 沉 淀内有 $\beta$ 折叠结构 ${ }^{[21]}$,这些 $\beta$ 折叠结构是与硫黄素 结合的关键分子基础。硫黄素可以和 $A \beta$ 中 $\beta$ 折叠 结构特异性结合, 在激发光下呈现绿色苂光。硫 磺素 $\mathrm{T}$ 染色结果显示, 中药地黄可以有效减少 $\mathrm{A} \beta$ 在脑内的沉积。相关研究表明,脑中约 $90 \%$ 的 $A \beta$ 通过血脑屏障转移人血, 并经过肝脏降解 ${ }^{[11]}$, 在 $A \beta$ 转运中起到重要作用。血脑屏障主要由脑微血 管内皮细胞及内皮细胞间的紧密连接蛋白、周细 胞、完整的基膜和胶质细胞足突组成 ${ }^{[22]}$ 。其中, 脑微血管内皮细胞是脑内与外周血之间进行物质 交换的主要场所, 是构成其基本结构和维持其功

表 2 各组大脑皮层和海马CA1区 $\beta$ 淀粉样蛋白沉积量和面积占比

Table 2 Amyloid $\beta$-protein positive areas and proportions in cortex and hippocampus CA1 area in each group

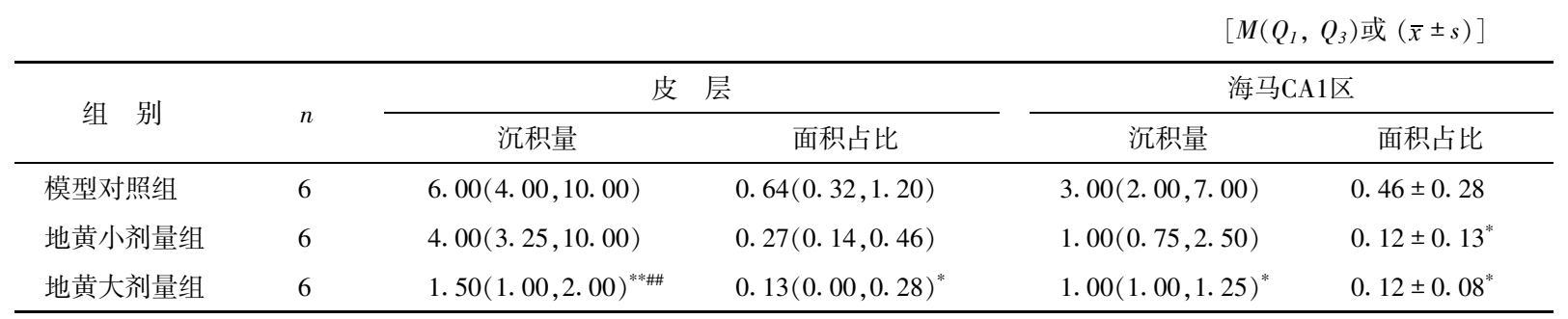

与模型对照组比较, ${ }^{*} P<0.05,{ }^{* *} P<0.01$; 与地黄小剂量组比较, ${ }^{\# \#} P<0.01$. 

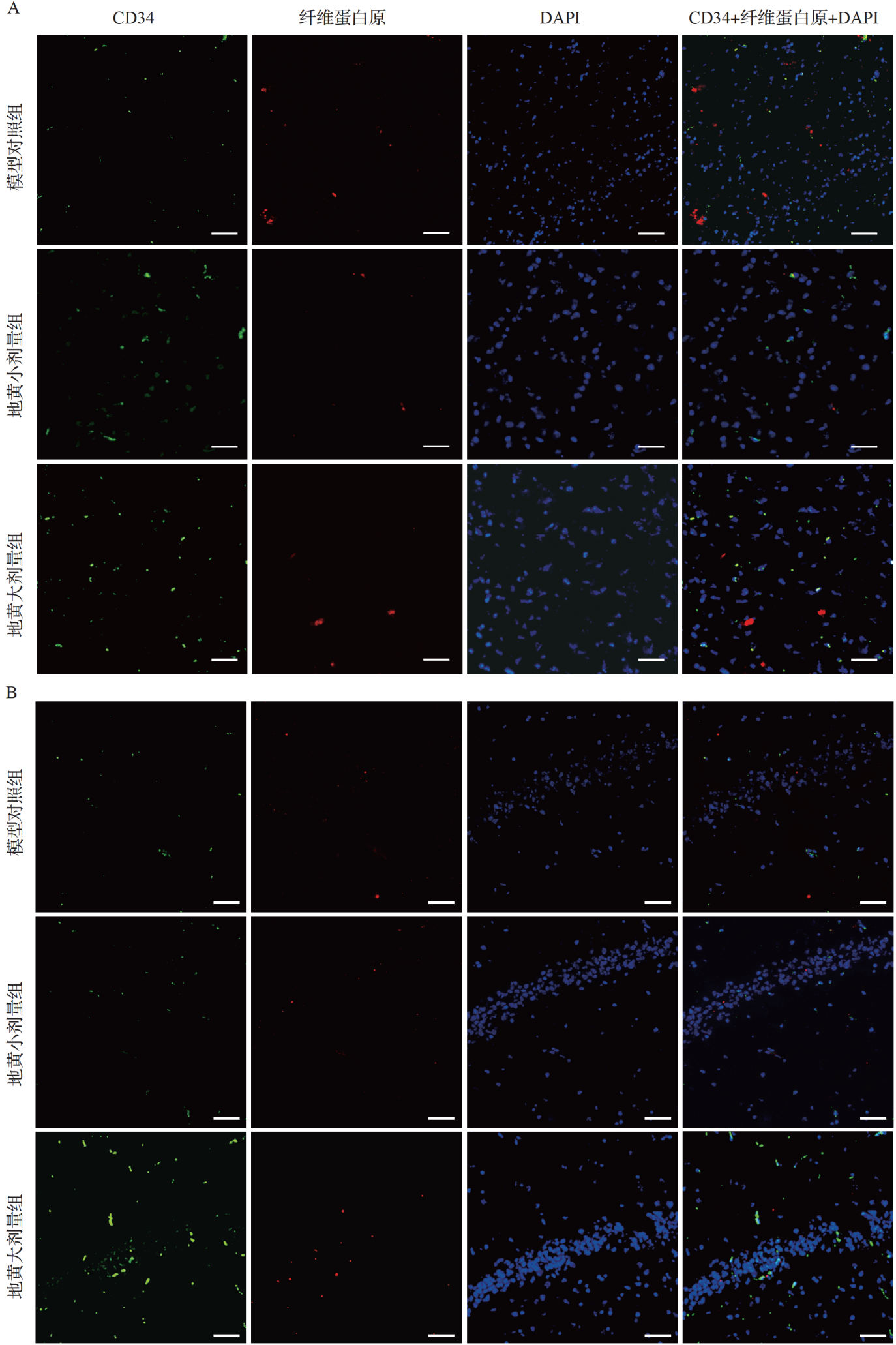

绿色苂光为CD34阳性表达, 红色苂光为纤维蛋白原阳性表达. A: 与模型对照组比较, 地黄小剂量组和地黄大剂量组皮层CD34 阳性面积明显增加, 纤维蛋白原阳性面积明显减少; 与地黄小剂量组比较, 地黄大剂量组皮层CD34面积明显增加. B : 与模型对照组 比较, 地黄小剂量组和地黄大剂量组海马CA1区CD34阳性面积增加, 纤维蛋白原阳性面积减少, 以地黄大剂量组更为显著. DAPI: 4', 6-二脒基-2-苯基吲哚. 标尺 $=100 \mu \mathrm{m}$.

图 2 各组大脑皮层和海马CA1区CD34、纤维蛋白原免疫菼光双重染色结果

Figure 2 Immunofluorescence double staining of CD34 and fibrinogen in cortex and hippocampus CA1 in each group 
表 3 各组大脑皮层和海马CA1区CD34和纤维蛋白原阳性面积占比比较

Table 3 CD34 and fibrinogen positive area proportion in cortex and hippocampus CA1 area in each group

\begin{tabular}{|c|c|c|c|c|c|}
\hline \multirow{2}{*}{ 组 别 } & \multirow{2}{*}{$n$} & \multicolumn{2}{|c|}{ 皮 层 } & \multicolumn{2}{|c|}{ 海马CA1区 } \\
\hline & & CD34 & 纤维蛋白原 & CD34 & 纤维蛋白原 \\
\hline 模型对照组 & 6 & $0.124(0.083,0.200)$ & $0.313(0.167,0.444)$ & $0.136(0.087,0.217)$ & $0.209 \pm 0.025$ \\
\hline 地黄小剂量组 & 6 & $0.295(0.197,0.358)^{*}$ & $0.111(0.067,0.146)^{* *}$ & $0.202(0.148,0.415)$ & $0.117 \pm 0.073$ \\
\hline 地黄大剂量组 & 6 & $0.431(0.324,0.551)^{* * \# \#}$ & $0.092(0.080,0.104)^{* *}$ & $0.442(0.313,0.665)^{*}$ & $0.110 \pm 0.043^{*}$ \\
\hline
\end{tabular}

与模型对照组比较, ${ }^{*} P<0.05,{ }^{* *} P<0.01 ;$ 与地黄小剂量组比较, ${ }^{*} P<0.01$.

能的最关键部分, 在整个血脑屏障中起着重要作 用 ${ }^{[23]}$ 。 $A \beta$ 通过损伤脑微血管内皮细胞与紧密连 接蛋白及相关转运蛋白的表达引起血脑屏障的 功能异常及其通透性增加 ${ }^{[24]}$ 。一方面, 高渗透性 的血脑屏障使得外周循环中的神经毒素、致病微 生物、炎症因子及免疫应答产物等进入脑内,引发 并加剧 $\mathrm{AD}$ 病情发展 ${ }^{[25]}$ 。另一方面, $\mathrm{AD}$ 患者脑内 $A \beta$ 可以介导血脑屏障上紧密连接蛋白及相关转运 蛋白的异常表达, 导致脑内 $\mathrm{A} \beta$ 跨血脑屏障清除率 大大降低, $A \beta$ 在脑内大量聚集, 使神经元及内皮细 胞损伤甚至调亡, 从而加剧 $\mathrm{AD}$ 病情 ${ }^{[26]}$ 。在血管 内皮细胞标记物中, CD34抗原作为一种高度糖基 化 I 型跨膜蛋白特异性最高, 是最敏感的内皮细 胞标记物 ${ }^{[27]}$ 。纤维蛋白原是人正常血浆蛋白成 分, 对细胞无毒副作用, 不受浓度、电荷及巨噬细 胞的影响,能更真实准确地反映血管的通透状 态 ${ }^{[28]}$,近年来作为内源性示踪剂被广泛采用。在 中枢神经系统中, 血脑屏障独特的屏障机制可以 严格调控血液中的蛋白进人神经系统, 正常情况 下中枢神经系统内检测不到纤维蛋白原的存在。 但在 $\mathrm{AD}$ 、缺血缺氧、放射性损伤等情况下血脑屏 障被破坏 ${ }^{[29]}$, 血液中可溶性纤维蛋白原则上可沉 积于脑组织中。本研究以 CD34标记微血管, 以纤 维蛋白原作为内源性示踪剂观察血脑屏障渗出情 况。结果显示, 中药地黄可以减轻血脑屏障的渗 出,对血脑屏障有一定的保护作用。

综上所述, 中药地黄特别是大剂量地黄可以 较好地维护脑内微血管的完整,降低血脑屏障的 通透性, 加大脑内 $\mathrm{A} \beta$ 清除, 改善 $\mathrm{AD}$ 模型小鼠的日 常活动能力和恐惧记忆功能。本研究为中药地黄 保护血脑屏障的体内机制提供了一定的实验依 据,可为补肾益精法治疗 $\mathrm{AD}$ 提供参考。

利益冲突 所有作者均声明不存在利益冲突

\section{参考文献}

[1] FAROUK F M, OOI L, LAW C S W, et al. Dual-targetdirected ligand displaying selective butyrylcholinesterase inhibitory and neurite promoting activities as a potential therapeutic for Alzheimer's disease $[J]$. Chemistry Select, 2020, 5(36): 11229-11236.

[2] 周仲英. 中医内科学 $[\mathrm{M}]$. 北京: 中国中医药出版社, 2003: 180 .

ZHOU Zhongying. Internal medicine of traditional Chinese medicine $[\mathrm{M}]$. Beijing: China Press of Traditional Chinese Medicine, 2003: 180. (in Chinese)

[3] 芦 锰, 周雨慧, 李晓宁, 等. 基于数据挖掘中医药治疗 阿尔茨海默病用药规律研究 $[\mathrm{J}]$. 中国中药杂志, 2021 , 46(6): 1558-1563.

LU Meng, ZHOU Yuhui, LI Xiaoning, et al. Research on regularity of traditional Chinese medicine in treatment of Alzheimer's disease based on data mining $[\mathrm{J}]$. China Journal of Chinese Materia Medica, 2021, 46(6): 1558-1563. (in Chinese)

[4] TROMPETERO A, GORDILLO A, DEL PILAR M C, et al. Alzheimer's disease and Parkinson's disease: a review of current treatment adopting a nanotechnology approach [J]. Curr Pharm Des, 2018, 24(1): 22-45.

５］王丹丹. “补肾益精方” 含药血清对血脑屏障的影响 及该方治疗轻度认知功能障碍的疗效评价 $[\mathrm{D}]$. 南京: 南京中医药大学, 2016.

WANG Dandan. Evaluate the effect of serum containing Bushenyijingfang on the blood-brain barrier and the clinical study of Bushenyijingfang on mild cognitive impairment [D ]. Nanjing: Nanjing University of Chinese Medicine, 2016. (in Chinese)

[6] 刘晨阳. 梓醇对纤维状 $A \beta_{1-42}$ 介导的体外血脑屏障的 损伤具有保护作用 $[D]$. 南京: 南京中医药大学, 2018. LIU Chenyang. Catalpol provides a protective effect on fibrillary $A \beta_{1-42}$-induced barrier disruption in an in vitro model of the blood-brain barrier[D]. Nanjing: Nanjing University of Chinese Medicine, 2018. (in Chinese)

[7] 陆韵薇, 于顾然, 符布清. 生地黄水煎剂对 $\mathrm{H}_{2} \mathrm{O}_{2}$ 诱导 的PC12 细胞凋亡的保护作用研究 $[\mathrm{J}]$. 江苏中医药, 2016, 48(10): 103-105.

LU Yunwei, YU Guran, FU Buqing. Protective effect of 
Radix Rehmanniae Recen water decoction on $\mathrm{H}_{2} \mathrm{O}_{2}-$ induced apoptosis of PC12 cells $[\mathrm{J}]$. Jiangsu Journal of Traditional Chinese Medicine, 2016, 48(10): 103105. (in Chinese)

[8] National Research Council (US), Committee for the Update of the Guide for the Care and Use of Laboratory Animals, Institute for Laboratory Animal Research (US), et al. Guide for the care and use of laboratory animals $[\mathrm{M}]$. 8th ed. Washington (DC): National Academies Press, 2011.

[9] 陈 奇. 中药药理研究方法学 $[M]$. 第3 版. 北京: 人 民卫生出版社, 2011: 1261-1263.

CHEN Qi. Research Methods in Pharmacology of Chinese Materia Medica $[\mathrm{M}]$. 3rd ed. Beijing: China Press of Traditional Chinese Medicine, 2011: 12611263. (in Chinese)

[10] WESSON D W, WILSON D A. Age and gene overexpression interact to abolish nesting behavior in Tg2576 amyloid precursor protein (APP) mice $[\mathrm{J}]$. Behav Brain Res, 2011, 216(1): 408-413.

[11] LEWARCH C L, HOEKSTRA H E. The evolution of nesting behaviour in Peromyscus mice[J]. Anim Behaviour, 2018, 139: 103-115.

[12] 崔 瑛. 怀庆熟地黄益智作用研究 [D]. 北京: 北京 中医药大学, 2002.

CUI Ying. Experimental study on mechanism of promoting intelligence with Huaiqing Shudihuang [D]. Beijing: Beijing University of Chinese Medicine, 2002. (in Chinese)

[13] KOOK S Y, SEOK HONG H, MOON M, et al. Disruption of blood-brain barrier in Alzheimer disease pathogenesis $[\mathrm{J} / \mathrm{OL}]$. Tissue Barriers, 2013, 1(2): e23993.

[14] WILCOCK D M, COLTON C A. Anti-amyloid- $\beta$ immunotherapy in alzheimer's disease: relevance of transgenic mouse studies to clinical trials $[\mathbf{J}]$. J Alzheimer Dis, 2008, 15(4): 555-569.

[15] SZCZYPKA M S, KWOK K, BROT M D, et al. Dopamine production in the caudate putamen restores feeding in dopamine-deficient mice $[\mathrm{J}]$. Neuron, 2001, 30(3): 819-828.

[16] DEACON R M, CROUCHER A, RAWLINS J N. Hippocampal cytotoxic lesion effects on speciestypical behaviours in mice $[\mathrm{J}]$. Behav Brain Res, 2002, 132(2): 203-213.

[17] UYS J D, STEIN D J, DANIELS W M, et al. Animal models of anxiety disorders $[\mathrm{J}]$. Curr Psychiatry Rep, 2003, 5(4): 274-281.

[18] 吴 江. 神经病学 $[\mathrm{M}]$. 北京: 人民卫生出版社, 2010: 331
WU Jiang. Neurology[M]. Beijing: People's Medical Publishing House, 2010: 331. (in Chinese)

[19] SELKOE D J, HARDY J. The amyloid hypothesis of Alzheimer's disease at 25 years $[J]$. EMBO Mol Med, 2016, 8(6): 595-608.

[20] HARDY J A, HIGGINS G A. Alzheimer's disease: the amyloid cascade hypothesis $[\mathrm{J}]$. Science, 1992, 256(5054): 184-185

[21] STROUD J C, LIU C, TENG P K, et al. Toxic fibrillar oligomers of amyloid- $\beta$ have cross $-\beta$ structure $[\mathrm{J}]$. Proc

Natl Acad Sci U S A, 2012, 109(20): 7717-7722.

[22] DANEMAN R, PRAT A. The blood-brain barrier[J]. Cold Spring Harb Perspect Biol, 2015, 7(1): a020412.

[23] 常 铮, 许继平. 脑血管内皮细胞与神经系统变性 病关系的研究进展 $[\mathrm{J}$ ]. 临床医学工程, 2010, 3(17): 151-153.

CHANG Zheng, XU Jiping. Progress in study on relationship between brain microvascular endothelial cells and nervous system degenerative diseases $[\mathrm{J}]$. Clinical Medicine \& Engineering, 2010, 3(17): 151 153. (in Chinese)

[24] YAMAZAKI Y, KANEKIYO T. Blood-brain barrier dysfunction and the pathogenesis of Alzheimer's disease[J]. Int J Mol Sci, 2017, 18(9): 1965.

[25] MONTAGNE A, BARNES S R, SWEENEY M D, et al. Blood-brain barrier breakdown in the aging human hippocampus[J]. Neuron, 2015, 85(2): 296-302.

[26] SAGARE A P, BELL R D, ZLOKOVIC B V. Neurovascular dysfunction and faulty amyloid $\beta$ peptide clearance in Alzheimer disease $[\mathrm{J}]$. Cold Spring Harb Perspect Med, 2012, 2(10): a011452.

[27] 柏树令, 赵 丹. CD34抗原的生物学特性及其临床 应用 $[\mathrm{J}]$. 解剖科学进展, $2005,11(1)$ : 54-56, 60 .

BAI Shuling, ZHAO Dan. Biological features of CD34 antigen and its clinical application $[\mathrm{J}]$. Progress of Anatomical Sciences, 2005, 11(1): 54-56, 60. (in Chinese)

[28] RYU J K, DAVALOS D, AKASSOGLOU K. Fibrinogen signal transduction in the nervous system $[\mathbf{J}]$. J Thromb Haemost, 2009, 7(Suppl 1): 151-154.

[29］田书娟，吴卫平. 纤维蛋白沉积与多发性硬化和周 围神经损伤关系研究现状 $[\mathrm{J}]$. 脑与神经疾病杂志, 2008, 16(2): 149-151.

TIAN Shujuan, WU Weiping. The relationship between fibrin deposition and peripheral nerve injury in multiple sclerosis $[\mathrm{J}]$. Journal of Brain and Nervous Diseases, 2008, 16(2): 149-151. (in Chinese)

$$
\text { [本文编辑 沈 敏 余 方] }
$$

